\title{
Monitoring of Toxic Gases and Land Slide Prevention using IoT
}

\author{
Joshi Gunjan Shailesh ${ }^{1}$, Dr. Prof. A.M. Deshmukh ${ }^{2}$ \\ M.E. Student, Dept of Electronics \& Telecommunication, Sinhgad College of Engineering, Pune, India ${ }^{1}$ \\ Professor, Dept of Electronics \& Telecommunication, Sinhgad College of Engineering, Pune, India ${ }^{2}$
}

\begin{abstract}
Mining activities releases toxic and very harmful gases. This causes a lots of pressure in the mining industry. Thus to increase the productivity and reduce the cost of mining along with the consideration of the safety of workers, an innovative approach is required. A real time monitoring system that using WSN, in which there are multiple sensors. This system monitors surrounding some environmental parameters such as moisture, toxic gases and land sliding with the help of two wireless sensor nodes using IoT. This system displays the parameters on the Monitor at the underground section, where sensor units will be install as well as on the monitoring unit. It will be helpful to all miners who present inside the mine to save their life before any causality occurs. An alarm will triggers when the sensor values crosses the threshold level. This system also stores all the data in the computer for future inspection.
\end{abstract}

Keywords: Internet of Things (IoT), Wireless sensor Network (WSN), Microcontroller, Raspberry pi.

\section{INTRODUCTION}

Underground mining opertion proves to be a risky as far as the health and the safety of the workers are conserned. These risks are belonged to different techniques used for extracting minerals. In underground mine, the ventilation systems are very critical to supply enough oxygen. So it is necessary to implement of mine monitoring systems for the safety purpose.

For some reason wired network is not possible and also it is time consuming. So it is essential to have a wireless sensor network mine monitoring system.

Wireless sensor network (WSNs) have earned a significant attention in current scenario. It is a special ad-hoc, selforganizing network that consists of large number of nodes arranged in a wide area.

Wireless sensor network mainly consist of sensor nodes responsible for sensing phenomenon which are responsible for collecting data from remote nodes and managing the network.

The Internet of Things (IoT) is a recent communication technology in which the objects will be equipped with transceivers,controllers for digital communication and also able to communicate with one another and with the users. IoT is based on WSN technology in which the systems are monitored over the internet. It can be operated over bluetooth, RF module,Wi-Fi module. WSN will be use to detect the toxic gases present in the mine and also will inform the dangerous situation in right time for the safety of miners. A real time monitoring system is develop to provide clear perspective of the underground mine. This sytem displays the parameters on the Monitor at the underground section, where the sensor unit and monitoring unit is installed. It will be helpful to all miners present inside the mine to save their life before any causality occurs. Alarm will trigger when sensor values crosses the threshold level. This system also stores all the data in the computer for future perspective.

\section{RELATED WORKS}

In underground mine, ventilation systems are critical to supply adequate oxygen, keeping up non-dangerous and nonlethal environments and an effective working mine. To monitor an underground mine, can help killing high hazard environments. Incorporating ventilation monitoring systems empowers a mine to insightfully roll out ventilation improvements in view of the far reaching information given by the monitoring systems. Sudden changes in the ventilation system are identified by the monitoring system, permitting quick move to be made. New and creating correspondence and following systems can be used to monitor mines more proficiently and transfer the information to the surface.

The progression of technology has allowed mine monitoring techniques to become more sophisticated, yet explosions in underground coal mines still occur. The safety issues of coal mines have gradually turned into a major concern for the society and nation. The occurrence of disasters in coal mines is mainly due to the harsh environment and variability of working conditions. So, it makes the implementation of mine monitoring systems essential for the safety purpose. Wired network systems used to be a trend for traditional coal mines, which have really played a significant role in 
safely production in coal mines. With the continuous enlargement of exploiting areas and depth expansion, laneways have become blind zones, where numerous unseen dangers are hidden out. Moreover, it is not possible there to lay expensive cables, which is also time consuming. So, it is essential to have a wireless sensor network mine monitoring system, which can be disposed in such mines in order to have a safe production within.

Wireless sensor networks (WSNs) have earned a significant worldwide attention in current scenario. A WSN is a special ad-hoc, multi-hop and self-organizing network that consists of a large number of nodes arranged in a wide area in order to monitor the phenomena of interest. Wireless sensor networks mainly consist of sensor nodes or motes responsible for sensing a phenomenon and base nodes, which are responsible for managing the network and collecting data from remote nodes.

These small sized sensors are quite inexpensive compared to traditional sensors and also require limited computing and processing resources. These sensor nodes can detect, measure and collect information from the environment and based on some local statistical decision process, they can convey the collected data to the control room.

It has three major advantages over wired monitoring network systems:

1. There is no need of cables to lay and easy installation in blind areas, reducing cost of the monitoring system. The number of nodes can be increased to eliminate blind areas. Also, it offers a general communication and allocation of the goal.

2. The dense nodes ensure the data acquisition with high accuracy and optimum data transmission, and further realization of real-time monitoring system for mine environment.

3. A little computing ability, storage capacity with data fusion of sensor nodes make them suitable for the remote monitoring system.

This paper proposes a Mine safety system which employs the integartion of Wireless sensor nodes(WSNs), Cloud Networking and IoT that provide the existance of toxic gases and Moisture of land in underground mine using sensors and microcontrollers and aware to the monitoring and sensor unit. Also the database present online with the main server for future inspection. The Proposed system supports more flexibility and security. This system is mainly consisting of three main components; the sensor nodes,Wi-Fi connection and a Raspberry-Pi Board. The Raspberry-Pi board is configured according to the service oriented architecture. The sensor unit senses the toxic gases and land moisture present in the underground mine and also in which direction the gases are spread with the help of wireless sensor nodes and upload the data using Microcontroller. Then the data is transmitted and then received by the Raspberry-Pi model where it is located in sensor and control unit in the underground mine. The data are uploaded using Wi-Fi to the backend server. At where the user can read the data for reference and also for future inspection.

\section{Advantages of the Proposed System:}

$>$ Detection of different toxic gases within mining environment.

$>$ Detection of Land sliding using IoT.

$>$ Develop the Technology to where the data is able to monitored all over the world.

$>$ Increase the Safety of Miners.

\section{IMPLEMENTATION}

In this System, for the monitoring of toxic gases and land sliding prevention using IoT for controlling of microcontroller based on the Raspberry-Pi board. Where the Raspberry-Pi is an open source which is used for software and hardware part on the board. As the communication between the Raspberry-Pi is wireless, the connectivity between them is established by the use of RF module and Bluetooth module. Gas and Moisture sensors are used to check the real environmental condition inside the mine. These Gas and Moisture sensors are connected with the perticular arduino uno board. These arduino uno collects data from the sensors. There are two wireless sensor node for collecting the data.

One node is used for collecting sensor data and the other node is used for in which direction the gases are spread. After collecting the data from the sensor, the node informed monitoring unit section under the mine. An alarm with the sensors are used for the purpose of security inside the mine, where if any leaking of toxic gases or possibility of land sliding in the mine, it will sets an alarm and will inform to the monitoring unit.

The systematise of the project implementation as of the Architecture of its implementation is given below:

After receiving the data to the microcontroller, the microcontroller transmit the data wirelessly and received by the receiver at the Raspberry pi model. Here the microcontroller used Bluetooth and RF module to transmit and receive data wirelessly. The microcontroller used Bluetooth and RF transmitter for each wireless sensor node and the other side Raspberry pi used Bluetooth and RF receiver to receive the data wirelessly. Raspberry-Pi board is paired with the Wi-Fi router or LAN cable for connecting the internet. The Data are uploaded to the server using MQTT protocol. MQTT is a public subscribe based light weight messaging protocol for use on the top of TCP/IP protocol. 


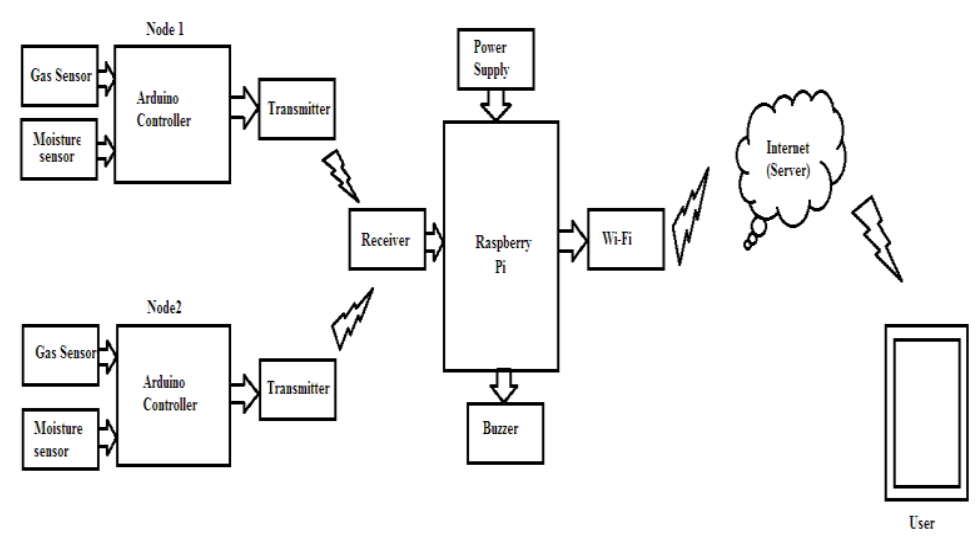

Fig.3.1 Architecture of the system

MQTT protocol contains MQTT Broker and MQTT Client. Where MQTT broker is responsible for receiving all the messages, Filtering them, decide who is interested in it and sending the message to the subscriber client. It is also responsible for network connection from clients and processes subscribe and unsubscribe requests from clients. The MQTT Client is a device that connected with the microcontroller. Here Raspberry pi is act as a MQTT client. MQTT client can be both publisher and subscriber at a same time. MQTT client is responsible for publish application message, subscribe to request application message, unsubscribe to remove a request for application messages and disconnect from the server. MQTT is best suited for IoT and M2M communication because of it small code footprint, lower bandwidth requirement and lower power consumption on device. Finally the data displays on the webpage. Thus user can read the data using web page and also use the data for future inspection.

\section{HARDWARE DESCRIPTION}

For this system, there are various hardwares are to be used, which are given below:

A. Carbon Monoxide Sensor(MQ3):

MQ-3 sensor consist of two parts. One is heating circuit and the other one is the signal output circuit. In which heating circuit is used for time control and signal output circuit is accurately respond changes of surface resistance of the sensor. This sensor senses the gases like methane(CH4), Carbon Monoxide(CO), Benzene, Hexene, Alcohol, LPG.

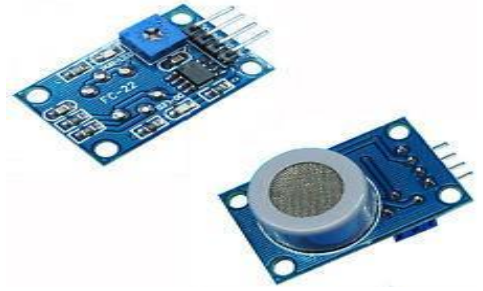

Fig.4.1 MQ-3 Sensor

The enveloped MQ-3 have six pins in which four of them are used to fetch the signals and other two are used for providing heating current.

\section{B. Mositure Sensor:}

This sensor can be used to test the moisture of soil, when the soil is having water particles, the module output is at high level, else the output is at low level. The operating voltage of this sensor is regulated 5v Dc.

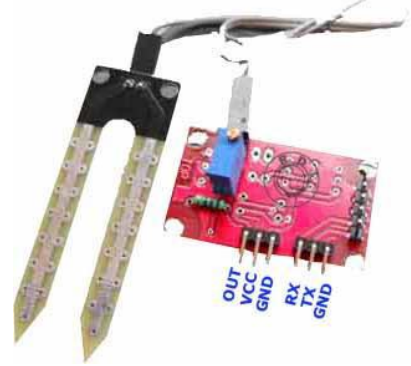

Fig. 4.2 Moisture Sensor 


\section{OPERATING ENVIRONMENT}

A. Hardware Required:

The monitoring system uses several components like Arduino uno, Raspberry pi 3 board, Rf module, MQ-3 Sensor, Bluetooth module, Moisture sensor, Buzzer etc.

B. Sofware Required:

The monitoring system uses several softwares like Raspberry OS, Python, Embedded C.

\section{SYSTEM OUTPUT}

The sensor unit senses the toxic gases and land moisture present in the underground mine with the help of wireless sensor nodes and upload the data using Microcontroller. The below table gives the values of gas sensors for normal and extreme condition. In normal condition, There is no toxic gases present in the mine and the value of gas sensor is 40 . In extreme condition, Toxic gases are present in the mine and the value of gas sensor is 345 .

6.1 Gas Sensor:

Table 1: Reading of Gas Sensor

\begin{tabular}{|l|l|l|}
\hline Operating Voltage & Condition & Output \\
\hline $5 \mathrm{~V}$ & Normal & 40 \\
\hline $5 \mathrm{~V}$ & Extreme & 345 \\
\hline
\end{tabular}

The threshold level for gas is above 300 and for moisture is $45-50$.

The below table gives the values of moisture sensors for normal and extreme condition. In normal condition, There is no moisture present in the soil. In extreme condition, There is a moisture present in the soil.

6.2 Moisture Sensor:

Table 2: Reading of Moisture Sensor

\begin{tabular}{|l|l|l|}
\hline Operating Voltage & Condition & Output \\
\hline $5 \mathrm{~V}$ & Normal & 5 \\
\hline $5 \mathrm{~V}$ & Extreme & 55 \\
\hline
\end{tabular}

In extreme condition whether the gas values or moisture values crosses the threshold level, the alarm will trigger and inform to the monitoring unit.

The output of this system is given as below:

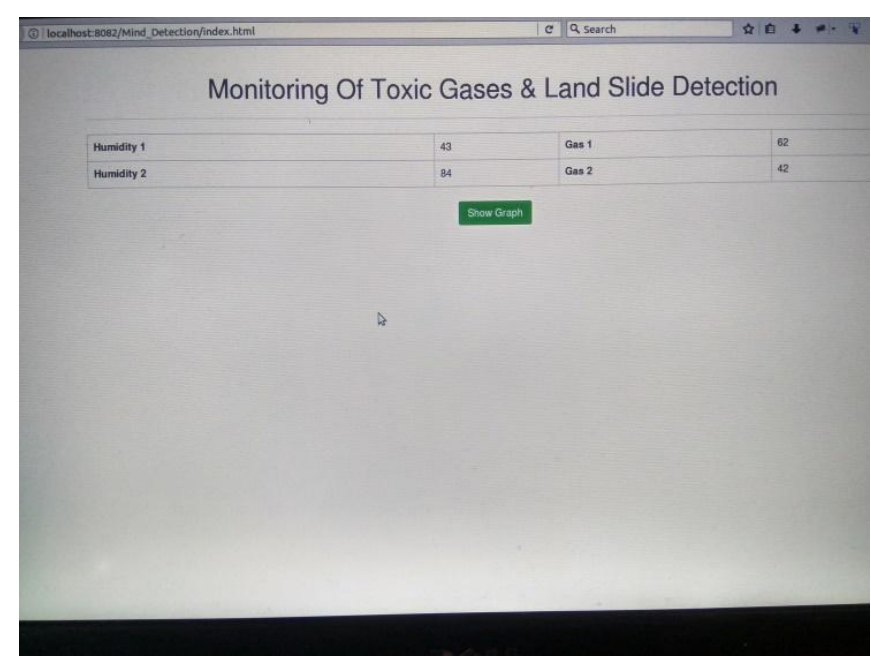

Fig. 5.1 Output of the system 


\section{CONCLUSION}

In this paper, The Study on real time monitoring of toxic gases and Moisture of Land present in underground mine analyze using wireless sensor network. This system is developed to provide clearer perspective of the mine and also display the parameters on the Monitor at the underground section. It will be helpful to all the miners who present inside the mine to save their life before any causality happens. An alarm will trigger when the sensor values crosses the threshold level. It also sends the sensor parameters to the cloud. This system will also stores the data in the computer for future inspection.

\section{REFERENCES}

1. Juan Felipe corso arias, Yeison julian camargo Barjas-International journal of advanced computer science and information technology(IJACSIT) vol.3,no.3,Page:327-343,Nov.2014.

2. Andrea Zanella, Nicoli Bui, Lorenzo Vangelista, Angelo Castellani, Michele Zorzi-Senior Member,IEEE-IEEE Internet Of Things For smart cities. Vol.1,no.1, Feb.2014.Page:1-4

3. Bo, C., Xiuqan, q., Budan, w., Xiaokun, w. et al. IEEE "Restful Web service Mashup based coal mine safety monitoring and control automation with wireless sensor network.”vol.2 Page:740-744,Aug.2012.

4. Dange , K.M., Patil, R.T. ,International journal of engineering science invention(IJESI) "Design of monitoring system for coal mine safety based on MSP430.” Vol.2.Page:14-19,July 2013.

5. Li, W., Li, Z., Liu, W., Wei, P. (2009),IEEE “The remote monitoring and analysis system of mine safety based on Virtual instrument."vol.2 Page:451-455.

6. J. Li, J. Wall and G. Platt, "Indoor air quality control of HVAC Syatem”, IEEE Int. conf. on Modelling, Identification and Control., page:756761, Aug 2010 .

7. He, X. Fan and D. Chai, "On-line control strategy of fresh air to meet the requirement of IAQ in office buildings," in IEEE Conf. on Industrial Electronics and application, page:845-848,June 2010.

8. Q. Lu, C. Wang and L. Shen,” Multi-zone particle model:The effect of ventilation on indoor concentration”, in IEEE Int. conf. on Computer Application and syaten Modelling, Page:503-507,Nov.2010.

9. Hasan omar al-sakran-king saud university,saudi arabia:International Journal Of Advanced Computer Science and Application(IJACSA) vol.6,no.2,Nov.2015.

10. V. Gokul, Sitaram Tadepalli (2016),IEEE "Implementation of a wifi based plug and sense dvice for dedicated air pollution monitoring using IOT.".vol.2. page: 355-358. 